\title{
Hybrid integration of fiber pigtailed silicon nitride microcavities with atom-chips for cavity QED applications
}

\author{
Paul E. Barclay, Kartik Srinivasan, Matthew Borselli, Thomas J. Johnson and \\ Oskar Painter \\ Thomas J. Watson, Sr. Laboratory of Applied Physics, California Institute of Technology, Pasadena, CA 91125, USA. \\ phone: (626) 395-6269, fax: (626) 795-7258, e-mail: pbarclay@caltech.edu \\ Benjamin Lev and Hideo Mabuchi \\ Norman Bridge Laboratory of Physics, California Institute of Technology, Pasadena, CA 91125, USA.
}

\begin{abstract}
Fiber coupled silicon nitride microcavities with $Q>3.6 \times 10^{6}$ and effective mode volume $<10(\lambda / n)^{3}$ at wavelengths resonant with alkali atom transitions are robustly integrated with magnetostatic atom-chips.

(C) 2006 Optical Society of America

OCIS codes: (230.5750) Resonators, (270.5580) Quantum electrodynamics
\end{abstract}

We demonstrate the suitability of silicon nitride $\left(\mathrm{SiN}_{x}\right)$ for high-Q microcavities at near-IR wavelengths necessary for cavity-QED experiments [1] with alkali atoms. Additionally, we demonstrate a robust mounting technique which enables the integration of a fiber coupled microcavity with a magnetostatic atom-chip delivery system [2]. The resulting hybrid atom-photon microchip can be installed in an ultra high vacuum system while maintaining highly efficient coupling between the microcavity and the outside world through a robust fiber optic input and output channel.
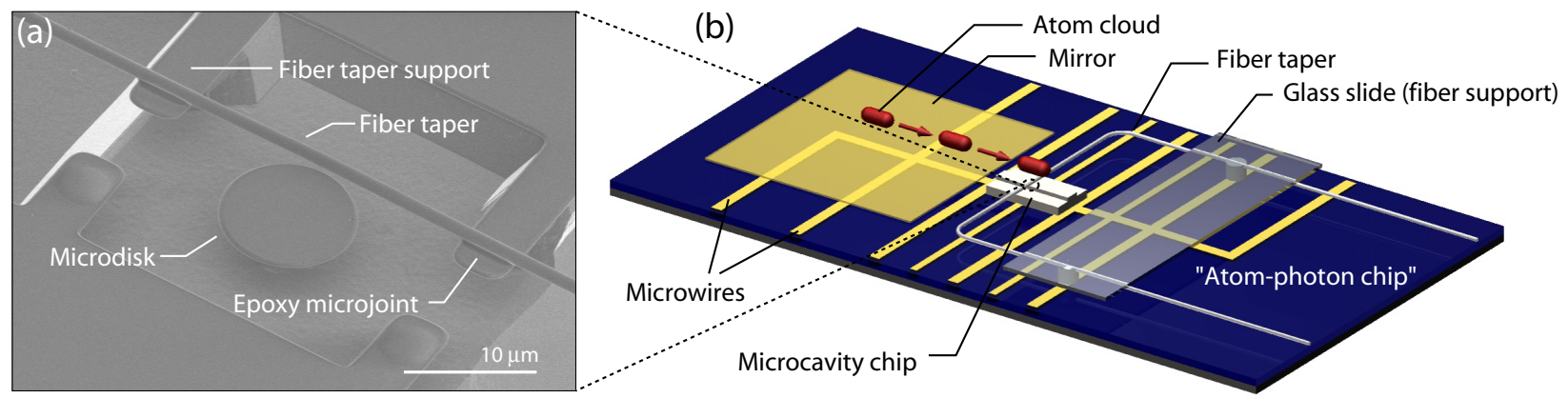

Fig. 1. (a) SEM image of a silicon nitride cavity robustly coupled to an optical fiber taper. The fiber taper is permanently aligned a few hundred nanometers from the microdisk circumference with epoxy microjoints to SiN supports. (b) Schematic of an atom-photon chip: a magnetostatic atom-chip integrated with a fiber coupled microcavity chip. The fiber taper pigtail is mounted to a low-profile glass slide which is bonded to the atom chip.

Employing fabrication techniques compatible with existing silicon [3] processing tools, $9 \mu \mathrm{m}$ and $11 \mu \mathrm{m}$ diameter microdisks where fabricated from $180 \mathrm{~nm}$ thick stoichiometric LPCVD silicon nitride deposited on a silicon substrate. A typical fabricated microdisk, as well as an optical fiber taper used for probing the optical modes of the device, is shown in Fig. 1(a). The fiber taper [4] provides highly efficient optical coupling to the microdisk [3], and after initial device characterization is permanently aligned with a suitable disk by UV epoxying the fiber taper to $\mathrm{SiN}_{x}$ supports lithographically defined near the microdisk, as shown in Fig. 1(a). The microdisk chip and fiber taper support are glued to a microfabricated atom-chip, as shown schematically in Fig. 1(b), and the assembly can be transported to and installed in a vacuum chamber without modifying the fiber-cavity alignment and coupling. Once in vacuum, the atom-chip can be used to magnetically transport laser-cooled atoms to the fiber-coupled microcavity.

The wavelength and quality factor of the microdisk modes were determined by measuring the transmission through the fiber taper as a function of wavelength, for varying taper position relative to the microdisk. Current work is focused on the $852 \mathrm{~nm}$ wavelength range overlapping the D1 and D2 transition wavelengths of cesium atoms used in atomic cavity QED experiments. However, it is expected that these devices will perform similarily at other alkali atom transition wavelengths [5]. Figure 2(a) shows typical transmission spectra through the fiber taper for wavelengths spanning a high-Q microdisk mode. The dips in transmission result from coherent coupling between the fiber taper and the microdisk modes, and the doublet structure is caused by surface roughness induced coupling between the degenerate 
clockwise and counterclockwise circulating whispering gallery modes of the microdisk [6,3]. The depth of the transmission dip can be tuned by varying the taper position relative to the microdisk. When the taper is positioned far from the microdisk, so that it induces negligible loss to the microdisk photon lifetime, the intrinsic photon loss rate can be determined from the linewidth of the dip. The resonance studied here has a linewidth $<0.26 \mathrm{pm}$, corresponding to a quality factor $Q>3.6 \times 10^{6}$. Finite element simulations of the disk predict an effective mode volume of $<10(\lambda / n)^{3}$. The corresponding photon decay rate $(\kappa / 2 \pi \sim 0.05 \mathrm{GHz})$ and cesium-photon coupling rate $\left(g_{0} / 2 \pi \sim 1.7 \mathrm{GHz}\right)$ place this cavity well in the regime of strong coupling [1].
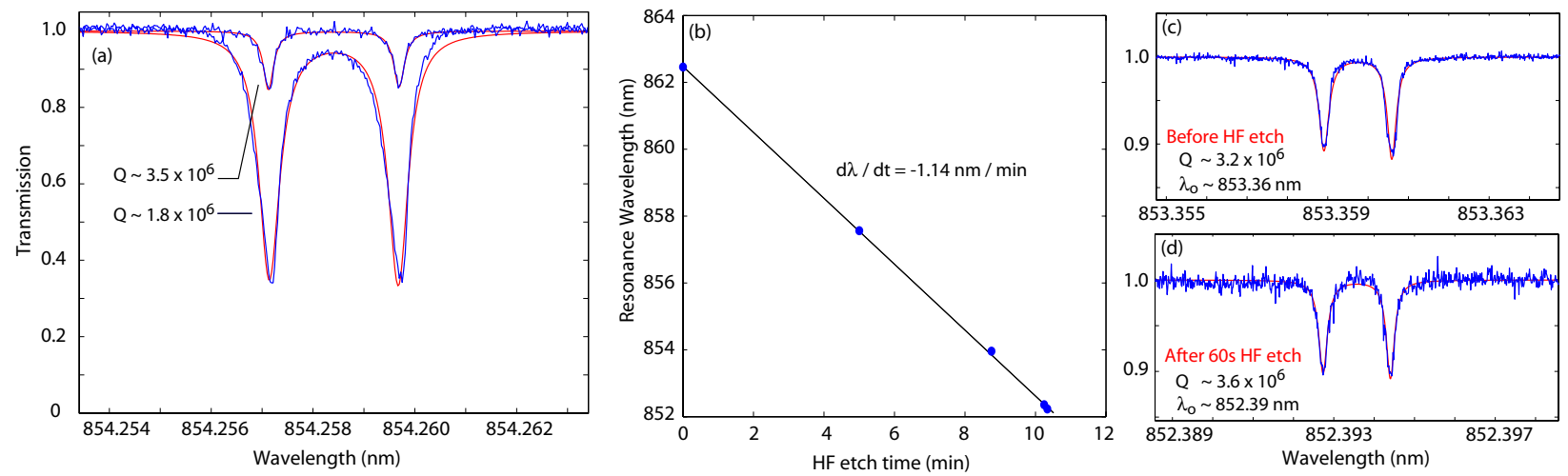

Fig. 2. (a) Typical measured high-Q microdisk mode lineshapes for two different taper-disk coupling strengths (i.e. taper positions). The transmission through the fiber taper is normalized to the transmission for zero coupling. The data (blue) is fit (red) using a model which takes into account coupling between the degenerate circulating modes of the microdisk. (b d) Tuning the microdisk resonance wavelength $\left(\lambda_{0}\right)$ using wet etching. A 20:1 solution of $\mathrm{H}_{2} 0: 49 \% \mathrm{HF}$ was used here. (b) High-Q resonance wavelength of a $11 \mu \mathrm{m}$ diameter microdisk as a function of $\mathrm{HF}$ etch time. The wet etch was interrupted at the time of each data point for the measurement of $\lambda_{b}$. Note that the final $\lambda_{0}$ is close to $852.34 \mathrm{~nm}$, the wavelength of the Cesium D2 line. (c) Mode lineshape before HF etch tuning. (d) Mode lineshape after a 60s HF etch. Note that the modal quality factor is not degraded by the HF tuning. A $9 \mu \mathrm{m}$ diameter disk was used in (c) and (d).

For microcavities to be useful in atomic cavity QED experiments, it is necessary to have the ability to finely control the cavity resonance wavelength so that it is close the atomic transition of interest. As shown in Fig. 2(b), beginning with a microdisk with a know resonance wavelength, we deterministically achieve this by dipping the microdisk in hydrofluoric acid (HF) solution. High quality $\mathrm{SiN}_{x}$ is etched very slowly by HF, resulting in a blue shift in the microdisk resonances. This technique provides $0.1 \mathrm{~nm}$ tuning accuracy without adversely affecting the device performance (Fig. 2(c-d)). The residual detuning from the atomic transition can be compensated for by heating or cooling the sample.

Our presentation will include recent measurements of the integrated atom-photon chip, including discussion of delivery of cold atoms to the microdisk and the behavior of the microdisk in ultra high vacuum.

\section{References}

[1] H. Mabuchi and A. C. Doherty, "Cavity Quantum Electrodynamics: Coherence in Context," Science 298, 1372-1377 (2002).

[2] J. Reichel, "Microchip traps and Bose-Einstein condensation," Appl. Phys. B 74, 469-487 (2002).

[3] M. Borselli, T. J. Johnson, and O. Painter, "Beyond the Rayleigh scattering limit in high-Q silicon microdisks: theory and experiment," Opt. Expr. 13, 1515-1530 (2005).

[4] J. Knight, G. Cheung, F. Jacques, and T. Birks, "Phase-matched excitation of whispering-gallery-mode resonances by a fiber taper," Opt. Lett. 22, 1129-1131 (1997).

[5] D. Gerace et al., "Wide-band transmittance of one-dimensional photonic crystals carved in Si3N4/SiO2 channel waveguides," Appl. Phys. Lett. 87, 211116 (2005).

[6] T. J. Kippenburg, S. M. Spillane, and K. J. Vahala, "Modal coupling in traveling-wave resonators," Opt. Lett. 27, 1669-1671 (2002). 